\title{
Dengue virus and Japanese encephalitis virus infection of the central nervous system share similar profiles of cytokine accumulation in cerebrospinal fluid
}

\author{
HAIPENG LII $I^{*}$, YUANYUAN LI ${ }^{I, 2^{*}}$, BO WEN ${ }^{I^{*}}$, JIAN ZHANG ${ }^{l}$, CHENGWU WANG ${ }^{l}$, ZHANYI SONG ${ }^{l}$, \\ SHUOCHI LI ${ }^{1}$, XIAOWANG QU ${ }^{1}$, RENBIN HUANG ${ }^{l}$, WENPEI LIU ${ }^{I^{*}}$ \\ ${ }^{1}$ Translational Medicine Institute, The First People's Hospital of Chenzhou, University of South China, Chenzhou, Hunan, China \\ ${ }^{2}$ Department of Neurology, The Third People's Hosptial of Yancheng, Yancheng, Jiangsu, China \\ *These authors made egnal contributions
}

\begin{abstract}
Dengue virus (DENV) and Japanese encephalitis virus (JEV) are two important pathogenic viruses that can cause severe encephalitis, which is accompanied by inflammatory cytokines. However, the inflammatory cytokine content of cerebrospinal fluid (CSF) in DENV and JEV infection of central nervous system are not sufficiently studied. To investigate cytokine levels in serum and CSF of hospitalised children with DENV and JEV infection of the central nervous system, a total of 183 hospitalised children with viral encephalitis-like syndrome were enrolled between May 2014 and April 2015 at the Children's Hospital of Chenzhou, Hunan, China. DENV and JEV infection was diagnosed by ELISA. Cytokine levels in the serum and CSF were measured by commercial ELISA kits. Twenty-nine (15.85\%) and $26(14.21 \%)$ DENV and JEV infections were detected in 183 patients with viral encephalitis-like syndrome, respectively. Higher granulocyte-macrophage colony-stimulating factor (GM-CSF) levels were detected in the serum of JEV infected patients than in DNEV patients $(p<0.05)$ or in healthy controls $(p<0.001)$, and levels of GM-CSF, interleukin $6(I L-6)$ and monocyte chemoattractant protein-1 (MCP-1) were higher in the CSF than serum in both DENV and JEV infection. Both DENV and JEV infection induced similar cytokine accumulation profiles in the CSF, which probably contributed to DENV-and JEV-induced immunopathogenesis.
\end{abstract}

Key words: dengue virus, Japanese encephalitis virus, central nervous system, cytokine.

(Cent Eur J Immunol 2017; 42 (2): 218-222)

\section{Introduction}

Each year 50 million people worldwide are infected with dengue virus (DENV) [1], which consists of four antigenically distinct serotypes (DENV-1, 2, 3, and 4) [2]. DENV infection causes dengue fever, dengue haemorrhagic fever (DHF), and dengue encephalitis in both children and adults [3, 4]. Dengue fever has recently become one of the most challenging public health problems in China. In June 2014, the most severe outbreak of dengue fever was reported in Guangzhou [5].

The pathophysiology of DF/DHF in humans likely involves the interplay of host and viral factors that influence disease severity and clinical outcome [6]. Previous studies have suggested that cytokines secreted by dengue-infected monocytes/macrophages influence DHF physiopathology.
Increased levels of interferon $\gamma($ IFN- $\gamma$ ), tumor necrosis factor $\alpha$ (TNF- $\alpha$ ), interleukin 6 (IL-6), and IL-8 were detected in adult patients with dengue fever [7, 8], and elevated levels of IL-6, IL-10, IFN- $\gamma$, macrophage migration inhibitory factor (MIF), and chemokine C-C motif ligand 4 (CCL-4) were associated with severity of dengue infection [9-11].

Some animal experiments have implicated direct neurovirulence of DENV infection in dengue encephalitis. The blood-brain barrier can be damaged by cytokines such as TNF-y and IFN- $\gamma[4,12]$. Other experiments indicated that elevated IFN- $\alpha$, TNF- $\alpha$, MIF, IL-8, IL-6, RANTES, cyclooxygenase 2 (Cox-2), IL-1 $\beta$, and MCP-1 levels were associated with poor outcomes in Japanese encephalitis (JE) patients [13-18].

Correspondence: Wenpei Liu or Renbin Miang, Translational Medicine Institute, The First People's Hospital of Chenzhou, University of South China, Chenzhou Human, China, No. 102 Luojiajing, Beihu District, Hunan, 423000, tel. 86-735-234 3902, fax 86-735-234 3902, China, e-mail: wenpeiliu_2008@hotmail.com (LWP), huang-renbin@163.com (HRB)

Submitted: 29.08.2016; Accepted: 6.09.2016 
However, the cytokine profiles of the cerebrospinal fluid (CSF) of patients with dengue encephalitis and JE remain to be established. This study aimed to investigate cytokine profiles in the cerebrospinal fluid (CSF) of children hospitalised with encephalitis-like syndrome and diagnosed with dengue infection of the central nervous system (CNS).

\section{Material and methods}

\section{Patients}

Between May 2014 and April 2015, 183 children (s 16 years of age) with encephalitis-like symptoms [19], admitted to The Children's Hospital of Chenzhou, Hunan Province, China, were included into this study. Blood and paired CSF samples were collected upon admission. Control blood samples were collected from 26 age-matched children who underwent surgery without current or recur- rent infectious diseases. The study protocol was approved by the Ethics Committee of the Hospital, and written consent was obtained from the parents of participants.

\section{Virus detection}

Dengue virus or Japanese encephalitis virus infection was confirmed by DENV IgM enzyme-linked immunosorbent assay (ELISA) (Virion/Serion, Germany) or JEV IgM ELISA assay (Boyan, Shanghai). The level of DENV RNA in each CSF sample from anti-DENV IgM-positive patients was tested by PCR, as described elsewhere [20]. Children with DENV and JEV co-infections were excluded from subsequent data analysis.

\section{Cytokine measurement}

We measured the level of IL-1 $\beta$, IL-2, IL-4, IL5, IL-6, IL-7, IL-8, IL-10, IL-12p70, IL-13, IL-17A,

Table 1. Clinical characteristics of hospitalized children with DENV encephalitis and JE

\begin{tabular}{|c|c|c|c|}
\hline Patient characteristics & DENV encephalitis $(n=29)$ & $\mathrm{JE}(n=26)$ & $P$ value \\
\hline \multicolumn{4}{|l|}{ Demographics } \\
\hline Male, $n(\%)$ & $16(55.2 \%)$ & $18(69.2 \%)$ & 0.284 \\
\hline Age, month (range) & $60.00(24.00-84.00)$ & $24.00(1.75-75.00)$ & 0.214 \\
\hline Setting (Rural/Urban) & $23 / 6$ & $21 / 5$ & 0.893 \\
\hline Course of illness, day rangea & $13.00(1150-17.50)$ & $14.50(9.75-22.25)$ & 0.532 \\
\hline \multicolumn{4}{|l|}{ Symptom } \\
\hline Peak body temperature $\left({ }^{\circ} \mathrm{C}\right)$ & $38.50(36.80-39.00)$ & $38.25(37.23-38.75)$ & 0.559 \\
\hline History of fever, $n(\%)$ & $21(72.4 \%)$ & $20(76.9 \%)$ & 0.702 \\
\hline Headache, $n(\%)$ & $15(51.7 \%)$ & $11(42.3 \%)$ & 0.485 \\
\hline Vomiting, $n(\%)$ & $13(44.8 \%)$ & $11(42.3 \%)$ & 0.851 \\
\hline Diarrhoea, $n(\%)$ & $0(0 \%)$ & $2(7.7 \%)$ & 0.219 \\
\hline Seizure, $n(\%)$ & $5(17.2 \%)$ & $3(11.5 \%)$ & 0.708 \\
\hline \multicolumn{4}{|l|}{ Sign } \\
\hline Neck stiffness, $n(\%)$ & $11(37.9 \%)$ & $6(23.1 \%)$ & 0.234 \\
\hline Kering sign, $n(\%)$ & $0(0 \%)$ & $1(3.8 \%)$ & 0.473 \\
\hline Brudzinski sign, $n(\%)$ & $0(0 \%)$ & $1(3.8 \%)$ & 0.473 \\
\hline Babinski sign, $n(\%)$ & $2(6.9 \%)$ & $2(7.7 \%)$ & 1.000 \\
\hline \multicolumn{4}{|l|}{ Image } \\
\hline Normal/abnormal & $11 / 5(n=16)$ & $8 / 11(n=19)$ & 0.115 \\
\hline Outcome at discharge & & & 0.492 \\
\hline Full recovery, $n(\%)$ & $27(93.1 \%)$ & $26(100 \%)$ & \\
\hline Mild neurological sequelae, $n(\%)$ & $2(6.9 \%)$ & $0(0 \%)$ & \\
\hline
\end{tabular}

${ }^{a}$ Course of illness is from the onset of symptom to disappear of symptom. DENV-dengue virus; JE-Japanese virus encephalitis 

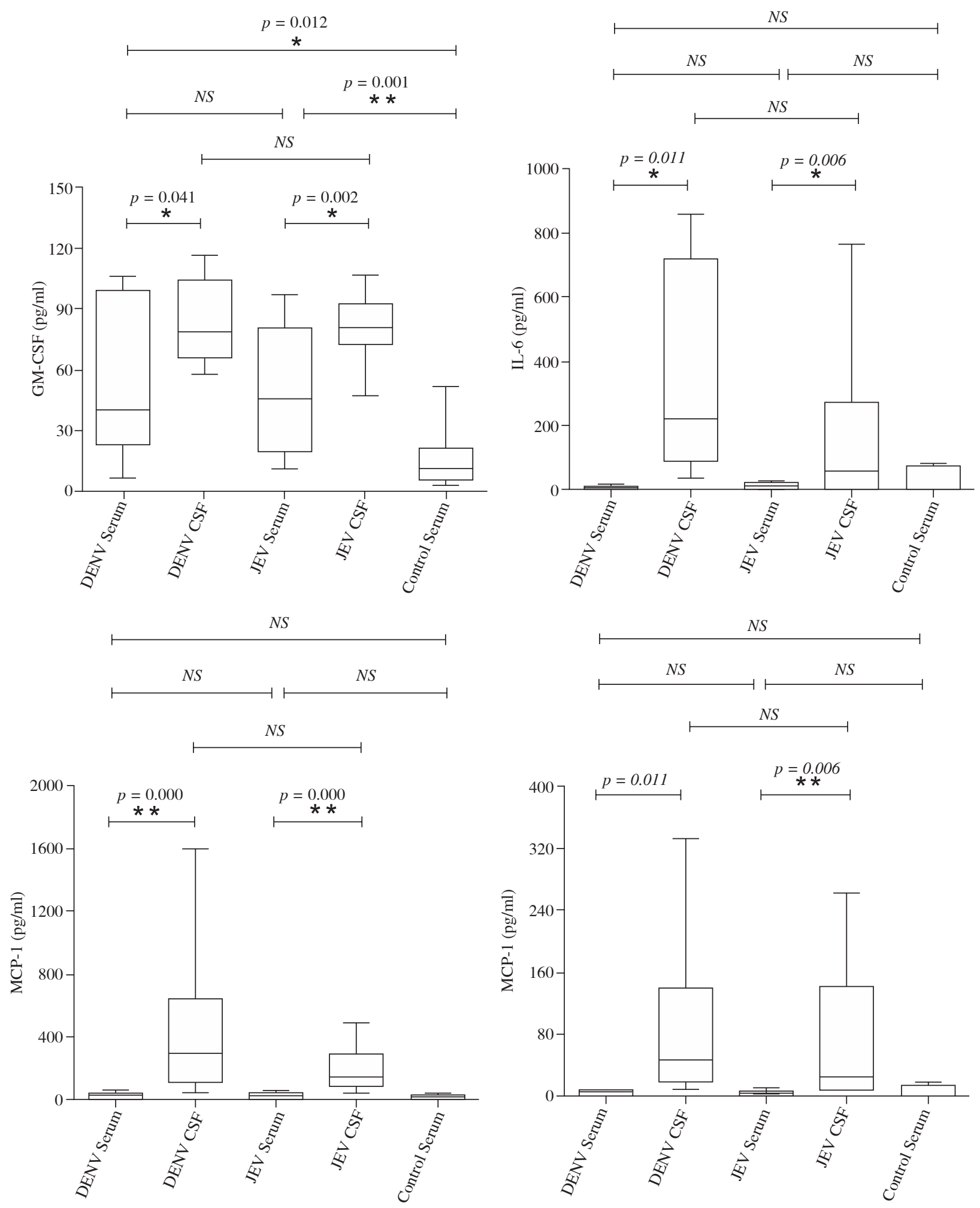

DENV - dengue virus; JEV - Japanese encephalitis virus; CSF - cerebrospinal fluid; NS - no significance; data were expressed as medians with the $10^{\text {th }}-90^{\text {th }}$ percentile range. Student's $t$ tests or Mann-Whitney U tests were performed. $P$ values were derived from a two-tailed test of significance: $* p \leq 0.05, * * p \leq 0.001$

Fig. 1. Expression level of GM-CSF (A), IL-6 (B), MCP-1 (C), and IL-8 (D) among patients with DENV infection of the central nervous system $(n=29)$, JEV infection $(n=26)$, and healthy control $(n=26)$ 
G-CSF, GM-CSF, IFN- $\gamma$, MCP-1, MIP-1 $\beta$, and TNF- $\alpha$ in the sera of healthy controls, and in the serum and CSF of DENV and JEV infected patients by Bio-Plex assay (BIORAD, USA).

\section{Statistical analyses}

The significance of differences between continuous variables was evaluated by Student's $t$ test or Mann-Whitney $U$ test, and the significance of differences between categorical variables was determined by $\chi^{2}$ test. Statistical analyses were carried out with SPSS 18.0 software. The result was considered significant when two-sided $p$ value was $<0.05$.

\section{Results and discussion}

Clinical characteristics of children with DENV and JEV infection of CNS

Of 183 hospitalised children with acute viral encephalitis-like symptoms, $29(15.85 \%)$ were diagnosed with DENV infection. The most common symptoms were vomiting $(44.8 \%)$, headache $(51.7 \%)$, and fever $(72.4 \%)$, their median age was 60 months, and $16(55.2 \%)$ were male (Table 1).

In addition, $26(14.21 \%)$ were diagnosed with JEV infection of the CNS. The most common symptoms were vomiting $(42.3 \%)$, headache $(42.3 \%)$, and fever $(76.9 \%)$, their median age was 24 months, and 18 (69.2\%) were male (Table 1).

Of the children hospitalised with acute viral encephalitis-like symptoms in this cohort, $15.85 \%$ and $14.21 \%$ were diagnosed with DENV and JEV, respectively. The higher incidence of DENV infection may reflect the spread of DENV infection following the June 2014 dengue fever outbreak in Guangdong province, which is adjacent to Chenzhou. The incidence of JEV infection was similar to previously published studies [21].

\section{Cytokine profiles in patients with dengue virus and Japanese encephalitis virus infection of CNS}

The level of granulocyte-macrophage colony-stimulating factor (GM-CSF) in the serum of patients with dengue encephalitis was significantly lower than that in JE patients (Fig. 1A), and the levels of IL-6, IL-8, MCP-1, and other cytokines did not differ significantly between the two groups (Fig. 1B-D).

The levels of GM-CSF, IL-6, and MCP-1 in the CSF of patients with dengue encephalitis and JE were significantly higher than in matched serum samples (Fig. 1A-C), whereas the levels of IL- 8 and other 13 cytokines (see in supplemental material 1 and 2) in the CSF did not differ significantly from matched serum samples from dengue encephalitis patients (Fig. 1D).

The CSF contained similar levels of cytokines in both dengue encephalitis and JE patients (Fig. 1A-D).
Interestingly, serum levels of GM-CSF were significantly lower in dengue encephalitis patients than in JE patients, but GM-CSF accumulated in CSF of patients with dengue encephalitis, exceeding levels in matched serum samples. GM-CSF has been reported to modulate immune responses in vivo [21], but the precise biological functions of elevated GM-CSF in the CSF warrants further investigation.

Interleukin 6 and MCP-1 accumulated in the CSF of both dengue encephalitis and JE patients, exceeding levels in matched serum samples, and IL-8 accumulated in the CSF of JE patients. Interleukin 6 is neurotrophic and neuroprotective and can increase blood-brain barrier permeability [17]. MCP-1 is the major monocyte chemoattractant produced during the inflammatory response in CNS infections. Interleukin 8 attracts and activates neutrophil leukocytes and is associated with blood-brain barrier breakdown [22]. Therefore, cytokine accumulation in the CSF may contribute to the immune response to dengue encephalitis and JE. The identification of similar CSF cytokine profiles in both dengue encephalitis and JE patients suggests that the cytokine response is non-specific and both dengue encephalitis and JE may share similar mechanisms of pathogenesis.

\section{Concluding remarks}

The results of the present study provide that DENV and JEV infection of the CNS was common among children, and induced expression of multiple cytokines. Several cytokines accumulated in the CSF, which may augment inflammation and other pathological changes in the infected brains. Effective treatment of dengue encephalitis and JE should take account of the high levels of inflammatory cytokines in affected brains, and aim to mitigate brain injury.

The authors thank all family members for enrolment in this study. The authors are appreciative of Department of Paediatrics of The First People's Hospital of Chenzhou for collecting samples. This work was supported by The First People's Hospital of Chenzhou, University of South China (Grant No. 2013-007, 2013-001, and 2014-003) The authors declare no conflict of interest.

\section{References}

1. Diaz-Quijano FA (2012): Dengue. N Engl J Med 367: 180; author reply 181.

2. Pok KY, Squires, RC, Tan LK, et al. (2015): First round of external quality assessment of dengue diagnostics in the WHO Western Pacific Region, 2013. Western Pac Surveill Response J 6: 73-81.

3. Kumar A, Gittens-St Hilair M, Jason V, et al. (2015): The clinical characteristics and outcome of children hospitalized 
with dengue in Barbados, an English Caribbean country. J Infect Dev Ctries 9: 394-401.

4. Matlani M, Chakravarti A, Rawal A, et al. (2009): Dengue encephalitis: an entity now common in dengue-prone regions. Trop Doct 39: 115-116.

5. Shen JC, Luo L, Li L, et al. (2015): The Impacts of Mosquito Density and Meteorological Factors on Dengue Fever Epidemics in Guangzhou, China, 2006-2014: a Time-series Analysis. Biomed Environ Sci 28: 321-329.

6. Tsai TT, Chuang YJ, Lin YS, et al. (2013): An emerging role for the anti-inflammatory cytokine interleukin-10 in dengue virus infection. J Biomed Sci 20: 40.

7. Raghupathy R, Chaturvedi UC, Al-Sayer H, et al. (1998): Elevated levels of IL-8 in dengue hemorrhagic fever. J Med Virol 56: 280-285.

8. Priyadarshini D, Gadia RR, Tripathy A, et al. (2010): Clinical findings and pro-inflammatory cytokines in dengue patients in Western India: a facility-based study. PLoS One 5: e8709.

9. Chen LC, Lei HY, Liu CC, et al. (2006): Correlation of serum levels of macrophage migration inhibitory factor with disease severity and clinical outcome in dengue patients. Am J Trop Med Hyg 74: 142-147.

10. Bozza FA, Cruz OG, Zagne SM, et al. (2008): Multiplex cytokine profile from dengue patients: MIP-1beta and IFN-gamma as predictive factors for severity. BMC Infect Dis 8: 86.

11. de-Oliveira-Pinto LM, Gandini M, Freitas LP, et al. (2012): Profile of circulating levels of IL-1Ra, CXCL10/IP-10, CCL4/ MIP-1beta and CCL2/MCP-1 in dengue fever and parvovirosis. Mem Inst Oswaldo Cruz 107: 48-56.

12. Amaral DC, Rachid MA, Vilela MC, et al. (2011): Intracerebral infection with dengue-3 virus induces meningoencephalitis and behavioral changes that precede lethality in mice. J Neuroinflammation 8: 23.

13. Kalita J, Srivastava R, Mishra MK, et al. (2010): Cytokines and chemokines in viral encephalitis: a clinicoradiological correlation. Neurosci Lett 473: 48-51.

14. Burke DS, Morill JC (1987): Levels of interferon in the plasma and cerebrospinal fluid of patients with acute Japanese encephalitis. J Infect Dis 155: 797-799.

15. Ravi V, Parida S, Desai A, et al. (1997): Correlation of tumor necrosis factor levels in the serum and cerebrospinal fluid with clinical outcome in Japanese encephalitis patients. J Med Virol 51: 132-136.

16. Singh A, Kulshreshtha R, Mathur A (2000): Secretion of the chemokine interleukin-8 during Japanese encephalitis virus infection. J Med Microbiol 49: 607-612.

17. Winter PM, Dung NM, Loan HT, et al. (2004): Proinflammatory cytokines and chemokines in humans with Japanese encephalitis. J Infect Dis 190: 1618-1626.

18. Ghoshal A, Das S, Ghosh S, et al. (2007): Proinflammatory mediators released by activated microglia induces neuronal death in Japanese encephalitis. Glia 55: 483-496.

19. Giri A, Arjyal A, Koirala S, et al. (2013): Aetiologies of central nervous system infections in adults in Kathmandu, Nepal: a prospective hospital-based study. Sci Rep 3: 2382.

20. Yavarian J, Gavvami N, Mamishi S (2014): Detection of human herpesvirus 6 in cerebrospinal fluid of children with possible encephalitis. Jundishapur J Microbiol 7: e11821.

21. Lanciotti RS, Calisher CH, Gubler DJ, et al. (1992): Rapid detection and typing of dengue viruses from clinical samples by using reverse transcriptase-polymerase chain reaction. J Clin Microbiol 30: 545-551.
22. Zhai Y, Zhou Y, Li X, et al. (2015): Immune-enhancing effect of nano-DNA vaccine encoding a gene of the prME protein of Japanese encephalitis virus and BALB/c mouse granulocyte-macrophage colony-stimulating factor. Mol Med Rep 12: 199-209.

23. Zhang JS, Zhao QM, Zuo SQ, et al. (2012): Cytokine and chemokine responses to Japanese encephalitis live attenuated vaccine in a human population. Int J Infect Dis 16: e285-288. 